\title{
ABORDAGEM FISIOTERAPÊUTICA NO ÂMBITO OCUPACIONAL - REVISÃO DA LITERATURA
}

\author{
PHYSIOTHERAPEUTIC APPROACH IN THE OCCUPATIONAL \\ SCOPE - LITERATURE REVIEW
}

\author{
Jefferson Júnio vidal Ramos ${ }^{1}$ \\ Sabrina Sampaio Santos ${ }^{2}$
}

RESUMO: O fisioterapeuta ocupacional vem tornando-se cada vez mais importante no meio industrial e comercial, visando sempre melhorar a qualidade de vida do trabalhador e prevenir lesões musculoesqueléticas. A questão norteadora deste trabalho aborta a importancia da fisioterapia no bem estar do trabalhador. Tendo como princípio a análise teórica de contextos impotantes que influênciam diretamentte na saúde e bem estar do colaborador devido atividades de muito esforço ou movimentos repetitivos, entendendo que isto causa frequentes acidentes e prejuízos aos trabalhadores e as empresas, trazemos essa análise. Os dados em estudo provêm de pesquisas de artigos cientificos realizadas nas plataforma CAPES, SciELO, LILACS e portal regional da BVS. Conclui-seque a atuação fisioterapeuta do trabalho dentro da empresa tem o objetivo de melhorar a qualidade de vida do trabalhador, resolve questões de ergonomia, implanta programas de cinesioterapia laboral, resolve questões judiciais relacionadas ao LER/DORT, e com isso o profissional tem melhor rendimento dentro da empresa, e um melhor bem estar físico e social fora dela.

Palavras-chave: Trabalhor. Fisioterapeuta. Qualidade de vida.

ABSTRACT: Occupational physiotherapists have become increasingly important in industrial and commercial environments, always aiming to improve workers' quality of life and prevent musculoskeletal injuries. The guiding question of this work aborts the importance of physiotherapy for the worker's well-being. Having as a principle the theoretical analysis of important contexts that directly influence the health and well-being of the employee due to high effort activities or repetitive movements, understanding that this causes frequent accidents and losses to workers and companies, we bring this analysis. The data under study come from research of scientific articles carried out on the CAPES platform, SciELO, LILACS and the regional portal of the VHL. It is concluded that the work physiotherapist's work

\footnotetext{
' Acadêmico do curso de Fisioterapia, Universidade da Amazônia (Unama) - PA, Email: jefferson.ifstm@hotmail.com.

${ }^{2}$ Acadêmica curso de Fisioterapia, Universidade da Amazõnia (Unama) - PA, Email: Sabrina-sophia@hotmail.com
} 
within the company aims to improve the worker's quality of life, resolve ergonomic issues, implement occupational kinesiotherapy programs, resolve legal issues related to LER/DORT, and with this the professional has better performance within the company, and better physical and social well-being outside it.

Keywords: Worker. Physical therapist. Quality of life.

\section{INTRODUÇÃO}

A ação do fisioterapeuta do trabalho se torna indispensável no ambiente industrial, devido ele ser o profissional com qualificações e habilidades para desenvolver atividades laborais que auxiliam na melhoria do tempo de trabalho e qualidade de vida, diminuindo dores e possíveis afastamentos trabalhistas. Neste artigo apresentam-se resultados de pesquisas e discursões sobre a temática já apresentada, tendo como propósito adiquerir e repassar conhecimentos sobre a importância de tal profissional mediante as atividades desenvolvidas nas empresas e campos de trabalho.

Aborda-se inicialmente a análise teórica de contextos impotantes que influênciam diretamentte na saúde e bem estar do trabalhador devido atividades de muito esforço ou movimentos repetitivos, entendendo que isto causa frequentes acidentes e prejuízos aos trabalhadores e as empresas.

A obtenção de dados deste estudo provêm de pesquisas de artigos cientificos realizadas nas plataforma CAPES, SciELO, LILACS e portal regional da BVS.

O referencial teórico e a triagem de estudos relacionados a temática permitem o esclarecimentos de diversos paradigmas sobre a atuação do fisioterapeuta do trabalho e sua ação na prevenção ou tratamento de lesões relacionadas a acidentes no âmbito trabalhista, com isso impõe uma melhor visão por partes de empresas e industras quanto a importância de tal profissional e seus benefícios mediante sua atuação efetiva.

\section{COMPETÊNCIAS DO FISIOTERAPEUTA DO TRABALHO}

A resolução no 43 de 18 de agosto de 20I do COFFITO - Art 3. Para o exercício da Especialidade Profissional de Fisioterapia do Trabalho é necessário o domínio das seguintes Grandes Áreas de Competência: [...] VIII - Avaliar a qualidade de vida no 
trabalho; e IX - Participar da elaboração de projetos e Programa de Qualidade de Vida e Saúde do Trabalhador; A partir da grande demanda de Fisioterapeutas, intervindo preventivamente e/ou terapeuticamente de forma significativa para a redução dos índices de doenças ocupacionais, e considerando que o fisioterapeuta é qualificado e legalmente habilitado para contribuir com suas ações para a prevenção, promoção e restauração da saúde do trabalhador, foi estabelecido em 2003, a Resolução 259 do COFFITO sobre a Fisioterapia do Trabalho.

Art. 6o A atuação do Fisioterapeuta do Trabalho se caracteriza pelo exercício profissional em todos os níveis de atenção à saúde, com ações de prevenção, promoção, proteção, rastreamento, educação, intervenção, recuperação e reabilitação do trabalhador, nos seguintes ambientes, entre outros: Hospitalar, ambulatorial, Domiciliar e Home Care, Públicos, Filantrópicos, Militares, Privados, Terceiro Setor, Rede pública em saúde do trabalhador, como, por exemplo, participar da rede pública de atenção e assistência em saúde do trabalhador como a RENAST (Rede Nacional de Atenção Integral à Saúde do Trabalhador), CEREST (Centro de Referência em Saúde do Trabalhador).

E ainda, a Classificação Brasileira de Ocupações (CBO) do Ministério do Trabalho e Emprego (MTE), define o fisioterapeuta como Cinesiólogo Fisioterapeuta e descreve que este profissional atende pacientes e clientes para prevenção, habilitação e reabilitação, realiza diagnóstico específico, desenvolvendo programas de prevenção, promoção de saúde e qualidade de vida. A aplicação dos princípios de ergonomia pode propiciar uma interação adequada e confortável do ser humano com os objetos que maneja e com o ambiente de trabalho, aumentando assim o seu desempenho.

A especialidade Fisioterapia do Trabalho, foi reconhecida pelo Conselho Federal de Fisioterapia (Coffito) a menos de ro anos. Com certeza, esse foi um grande passo dado pela área na ampliação da atuação profissional, assim, como mais recentemente a definição das atribuições do profissional (COFFITO, 2016).

Muito embora, tenhamos que reconhecer que o Fisioterapeuta reúne expertises para atuação na área de Saúde do Trabalhador e Ergonomia, já a partir de sua formação de 
graduação, pois possui grande capacidade de integrar diversos temas tais como biomecânica, educação em saúde. O crescimento da atuação do Fisioterapeuta em Saúde do Trabalhador no Brasil, com certeza se dará ainda mais com os avanços científicos na área, reforçam a necessidade do conhecimento da atuação desse profissional para que as necessidades observadas possam integrar as equipes que atuam em Saúde do Trabalhador, para contribuir com a agenda nacional no âmbito da assistência e das pesquisas (PADULA RS, et al, 2016)

\section{AÇÃO DO FISIOTERAPEUTA FRENTE A RISCOS E ACIDENTES ERGONÔMICOS}

A Organização Internacional do Trabalho (OIT) define ergonomia como um conjunto de ciências e tecnologias que procuram o ajuste entre o homem e o trabalho, tendo como resultado a eficiência humana e o bem estar do funcionário.

A ergonomia é a interação entre o homem e o trabalho, ou seja, é o modo com que o homem se relaciona com o ambiente físico e com os métodos utilizados para projetar e controlar o trabalho nesse ambiente (LIDA, 2003). Sendo assim, a ergonomia pesquisa as características físicas, psicofisiológicas e sociais no trabalho, incluindo o sexo, a idade, a motivação, o salário, a comunicação, a luz, os ruídos, a disposição mobiliária e muitos outros fatores (CHEREM E MAGAJEWSKI, 2003).

Segundo o relato de Carvalho e Ferreira (2006), a ergonomia teve sua origem formal durante a Segunda Guerra Mundial, quando instrumentos bélicos foram adaptados aos seus operadores e as condições desfavoráveis das atividades realizadas, estas modificações bem sucedidas deram origem, posteriormente, a Ergonomics Research Society, fundada em 1949, na Inglaterra. No entanto, o termo ergonomia já havia sido citado pelo polonês Woitej Yastembowsky, em 1857, no seu artigo intitulado "Ensaios de ergonomia ou ciência do trabalho, baseadas nas leis objetivas da ciência sobre a natureza" (LIDA, 2003).

Os riscos ergonômicos na saúde do trabalhador são provenientes da movimentação e posturas inadequadas, transporte de equipamentos e em atividades de organização e assistência. Além disso, os trabalhadores realizam rodízios de turnos e trabalho noturno. 
Essas ações causam à saúde dos trabalhadores problemas de postura, fadiga, hérnias, fraturas, torções, contusões, lombalgias e varizes. Sendo assim, a ação do fisioterapeuta do trabalho se torna imprescindível no ambiente de trabalho devido ele ser o profissional com qualificações e habilidades para desenvolver estudo científico das relações entre homem e máquina, visando uma segurança e eficientes ideais no modo como um e outra interagem, otimizando as condições de trabalho humano por meio de métodos e de novas tecnologias (Zapparoli AS, 2015).

Com a intervenção do fisioterapeuta do trabalho vários benefícios podem ser mencionar como exemplo a ginástica laboral, que proporciona tanto para o trabalhador, quanto para a empresa, resultados bem satisfatórios. Além de prevenir a LER/DORT, ela tem apresentado resultados mais rápidos e diretos como a melhora do relacionamento interpessoal e o alívio das dores corporais. Neste sentido, a implantação de um programa de ginástica laboral busca despertar nos trabalhadores a necessidade de mudanças do estilo de vida e não apenas de alteração nos momentos de ginástica orientada dentro da empresa. No ponto de vista empresarial, desenvolver ações de promoção em saúde e da qualidade de vida para os trabalhadores representa um investimento com retorno garantido a médio e longo prazo. O fisioterapeuta do trabalho avalia, previne e trata distúrbios ou lesões decorrentes das atividades no trabalho, realizando o estudo ergonômico do trabalho junto à equipe de saúde e segurança do trabalho, profere palestras de conscientização, capacitação e treinamento preventivo de doenças ocupacionais, realiza avaliação postural dos trabalhadores e análise biomecânica das tarefas nos postos de trabalho, desenvolver programas de ginástica laboral e é responsável pelo programa de tratamento ambulatorial de queixas músculo-esqueléticas com a utilização de todos os recursos fisioterapêuticos disponíveis através de um ambulatório que pode ser localizado dentro da própria empresa ou não (Paula A, et al, 2or6).

\section{INTERVENÇÃO FISIOTERAPÊUTICA EM DOÊNÇAS OSTEOMUSCULARES RELACIONADAS AO TRBALHO}


Os distúrbios osteomusculares relacionados ao trabalho (DORT) são afecções de músculos, tendões, sinóvias, nervos, fáscias e/ou ligamentos, isoladas ou combinadas, com ou sem degeneração de tecidos. Estas lesões atingem em maiores proporções os membros superiores, a região escapular e a região cervical, sendo sua origem ocupacional, decorrendo (de forma combinada ou não) do uso repetido ou forçado de grupos musculares e da manutenção de postura inadequada.( Oliveira RMR, 200I).

As estruturas músculo-esqueléticas passam a ser alvo frequente destas agressões independentemente do tipo de atividade ou do produto fabricado, do processo e organização do trabalho. Nesse contexto, os DORT têm se constituído em grande problema da Saúde Pública em muitos dos países industrializados. Essas doenças representam mais da metade das doenças ocupacionais, contabilizando em 20oI, segundo o Centro de Estudos da Saúde do Trabalhador (CESAT), 65\% dos casos de diagnósticos de doenças ocupacionais (Santos APA, et al, 2008).

Muitos colaboradores utilizam pouca força na realização de suas atividades, porém o que sobrecarrega são os movimentos repetitivos por amplos períodos de tempo, de maneira estática forçando o mesmo grupo muscular, levando a disfunções osteomusculares de trabalho (MEDEIROS; SEGATTO, 2012).

A DORT é considerada uma síndrome de origem ocupacional, cuja terminologia não é consensual, e até o momento é inexistente um diagnóstico desse problema no Código Internacional das Doenças (CID-Io), todavia, sua utilização é altamente divulgada na sociedade, como um todo em razão dos males provocados aos trabalhadores e ao grande impacto social (MORAES; BASTOS, et al, 2017).

O tratamento preventivo consiste na conscientização com campanhas, um local de trabalho ergonomicamente apropriado, com pausas e ginástica laboral. $\mathrm{O}$ tratamento clínico se dá em procedimentos médicos, com remédios antiinflamatórios e fisioterápicos, e quando o caso está em estágios avançados, o funcionário pode ser realocado e, até mesmo se distanciar do trabalho. A fisioterapia consiste em tratar os sinais e sintomas de algia, edema, diminuição de força e encurtamento, hipoestesia e hiperestesias. A conduta 
fisioterapêutica trata o quadro álgico com crioterapia, bandagens e TENS, o edema com ultrassom e massoterapia, a cinesioterapia trabalha com FES, movimentos ativos e ativos resistidos, e a sensibilidade pode ser tratada com chumaços de algodão, buchas e escovas (FERNANDES, 2016).

\section{CONDUTA FISIOTERAPÊUTICA NA PREVENÇÃO E TRATAMENTO DA LOMBALGIA OCUPACIONAL}

A Classificação Internacional de Comprometimentos, Incapacidades e Deficiências da Organização Mundial de Saúde reconhece a lombalgia como um comprometimento que revela perda ou anormalidade da estrutura da coluna lombar de etiologia psicológica, fisiológica ou anatômica ou, ainda, uma deficiência que traduz uma desvantagem que limita ou impede o desempenho pleno de atividades físicas. Ainda sob a perspectiva dessa classificação, a lombalgia pode evidenciar síndromes de uso excessivo, compressivas ou posturais, relacionadas a desequilíbrios musculares, fraqueza muscular, diminuição na amplitude ou na coordenação de movimentos, aumento de fadiga e instabilidade de tronco (World Health Organization, 1980).

Os fatores causais mais diretamente relacionados com as lombalgias ocupacionais são os mecânicos, os posturais, os traumáticos e os psicossociais. A idade, a postura e a fadig a no trabalho são consideradas como fatores contribuintes para a elevada percentagem de recidiva da dor lombar. O trabalho sentado por longas horas, o trabalho pesado, o levantamento de peso, a falta de exercícios físicos e os problemas psicológicos representam alguns dos principais fatores que contribuem para a cronicidade da dor lombar. Queixas frequentes de dor na coluna lombar estão associadas à tensão da musculatura paravertebral decorrente de posturas incômodas e da degeneração precoce dos discos intervertebrais pelo excesso de esforço físico. Acredita-se que muitos casos de lombalgia se devem a pressões incomuns sobre os músculos e os ligamentos que suportam a coluna vertebral (Andersson GBJ, 1999).

A dor sofre variações até na maneira de como o indivíduo relata, pois há relacionamento com inúmeros fatores pessoais tais como: sexo, idade, 
personalidade, herança étnica cultural, necessidades comportamentais e experiências dolorosas pregressas (CIENA et al, 2008 citado por GODINHO et al, 20II).

São considerados como fatores de risco para lombalgia ocupacional os traumas cumulativos, as atividades dinâmicas relacionadas com movimentos de flexão e rotação do tronco, o trabalho físico pesado, o agachamento, os macro-traumas, o levantamento ou carregamento de cargas, a exposição a longas jornadas de trabalho sem pausas, as vibrações de corpo inteiro e a adoção de posturas estáticas e inadequadas (Da Silva MC et al, 2006).

A eliminação dos fatores de risco, a terapia medicamentosa, a fisioterapia e a reeducação do paciente são os alicerces para o tratamento da dor lombar ocupacional. No que se refere à fisioterapia, não foram encontradas evidências científicas, até o momento, de que eletroterapias com calor local ou estimulação elétrica tenham relevância comprovada no tratamento da dor lombar crônica. São os exercícios físicos orientados que guardam relevância no seu tratamento. Embora a maioria dos episódios de lombalgia seja autolimitada, devem ser tratados prontamente e de forma eficaz, a fim de se prevenir a cronificação. Depois de afastadas as causas específicas, o tratamento deve ser centrado no controle sintomático da dor para propiciar a recuperação funcional no período mais breve possível (Fernandes AR et al, 2004).

\section{TRATAMENTO FISIOTERAPÊUTICA EM HÉRNIA DISCAL OCUPACIONAL}

A hérnia de disco, patologia bastante frequente nos dias atuais, é causada por uma lesão dos discos que compõem a coluna vertebral. Consiste em uma doença crônicodegenerativa da coluna, sendo atualmente considerada uma síndrome multifatorial com grandes impactos econômicos e emocionais de alta prevalência, podendo afastar indivíduos economicamente ativos de suas atividades sociais e laboraisı . No Brasil, as lombalgias têm se tornado a $\mathrm{I}^{\underline{a}}$ causa de pagamento de auxíliodoença e a $3^{\underline{a}}$ causa de aposentadoria por invalidez, gerando repercussões sobre a economia (Fernandes RCP, 1994).

A hérnia de disco é causada pelo processo de protrusão do disco intervertebral, por ruptura de suas fibras. Com isso, há o risco de pressionar as raízes nervosas no canal vertebral ou infeccioná-las ou inflamálas. Conforme a localização em que se estabelecem, 
causam os sintomas com dores. Seus sintomas dependem da localização, do tamanho, do tipo e do grau de envolvimento radicular (Santos M, 2003).

As hérnias podem ser assintomáticas, quando a herniação se direciona para o centro dos corpos vertebrais que delimitam o disco, ou sintomáticas quando vai para dentro do canal vertebral, comprimindo terminações e raízes nervosas. Quando sintomática, a dor lombar, um dos principais sintomas da hérnia de disco lombar, é caracterizada por uma dor inicialmente na região lombar, que pode irradiar-se para nádegas, coxas e joelhos, podendo ser uma dor aguda, que piora com o esforço físico, o que geralmente ocorre em jovens, ou permanente, mas não muito intensa, o que ocorre principalmente em idosos (Ortiz J, Abreu AD, 200o).

Dentre algumas medidas eficazes na prevenção da hérnia de disco, tem-se manter a postura ereta, transportar carga junto ao corpo, evitar torções da coluna, corrigir a postura no trabalho, uso de sapatos confortáveis, perder peso, praticar atividades físicas, fazer alongamentos e aquecimento antes de fazer qualquer esforço com a coluna. $\mathrm{O}$ diagnóstico pode ser feito clinicamente e por exame neurológico. Exames como o raio X, tomografia e ressonância magnética ajudam a determinar o tamanho da lesão e em que exata região da coluna está localizada (Vialle LR et al, 20ro).

Atualmente, a hérnia lombar é o diagnóstico mais comum dentre todas as alterações degenerativas dessa região e a principal causa de cirurgia de coluna. Fatores como o maior acesso a cuidados médicos, precocidade na solicitação de exames de imagem e segurança dos procedimentos cirúrgicos levam a altas taxas da cirurgia, condição que é geralmente autolimitada (MACHADO et al, 2013).

O tratamento fisioterapêutico na hérnia de disco é conservador e primeiramente deve ser realizada uma avaliação. A anamnese visa detectar o estágio que o paciente se encontra, a intensidade da dor, tipo de laser e de trabalho, o paciente irá descrever toda a sua história pregressa. Avaliação neurológica ajuda na identificação do tipo de marcha, força, tônus muscular, diminuição ou aumento dos reflexos, coordenação e sensibilidade. Avaliação postural auxilia na detecção de alterações visíveis da coluna. No exame físico serão realizados alguns testes ortopédicos essenciais, o sinal de Laségue provoca tensão sobre o nervo ciático, se o paciente referir dor, o sinal é positivo. Os testes de elevação de perna e de elevação de perna retificado são significantes para o diagnostico de hérnia de 
disco lombar.

A fisioterapia tem como objetivo: controlar a dor, reduzir espasmos, reduzir parestesias e contraturas articulares, restabelecer o equilíbrio da coluna, fortalecer a 4 musculatura paravertebral e acessórios. Os recursos fisioterapêuticos utilizados incluem a crioterapia, o TENS que estimula as fibras nervosas A-alfa de condução rápida tendo efeito analgésico e reduzindo espasmos, ultrassom terapêutico que gera o aumento do fluxo sanguíneo, tração manual e terapia manual. As técnicas de mobilização neural têm sido utilizadas na recuperação de pacientes com distúrbios musculoesqueléticos (SOARES, 2010).

\section{METODOLOGIA}

O estudo apresentado incide na análise teórica a cerca de contextos impotantes que influênciam diretamentte na saúde e bem estar do trabalhador devido atividades de muito esforço ou movimentos repetitivos. Tendo em vista a importância da fisioterapia no processo de prevenção ou tratamento de doênças ocupacionais de competências fisioterapêuticas.

De caráter descritivo, a revisão baseou-se em uma abordagem qualitativa para para sua construção, na qual foi realizado triagem efetiva na busca de artigos originais e cientificos para elaboração, tendo como base de pesquisa plataformas importantes e confiáveis como, CAPES, SciELO, LILACS e portal regional da BVS.

No primeira momento, foi realizado a escolha do tema, visto a importância de tal temática, após isso, a realização da triagem de artigo que foram de primordial importância para a contrução do trabalho e para aprimoramento de conhecimento pessoal e profissional.

No segundo momento, foi dado andamento na realização do estudo, construção da estrutura de acordo com as normais exigidas, e elaboração de conteúdos necessários para real efetivação do trabalho.

A opção pelo método de revisão da lieratura, se deu pela importância de informações cocreta de cunho ciêntifico que justificasse a temática estudada, auxiliando 
na elaboração do trabalho e dando veracidade as informações submetidas a este estudo.

\section{RESULTADOS}

Como resultados é possível ênfatizar que o profissional especialista em fisioterapia do trabalho torna-se cada vez mais peça fundamental no meio industrial devido atuar principalmente em áreas como a ergonomia e biomecânica, juntamente a uma equipe multidisciplinar, sua atuação visa melhorar a qualidade de vida do trabalhador e prevenir lesões músculo esqueléticas. O resultado desta intervenção é um melhora no desempenho e na produtividade no trabalho (BAÚ, 2002).

A regulamentação do fisioterapeuta nesta área possui uma importância singular, devido ser um membro da equipe de saúde com sólida formação científica, que atua no desenvolvimento de técnicas de prevenção, avaliação, tratamento e reabilitação, que utilizando programas de orientações e promoção em saúde atua nos três níveis de atenção (TALO et al, 1995).

Tsuchiya et al (2009) coloca que o ambiente de trabalho necessidade da atuação do fisioterapeuta, profissional apto a atuar preventivamente, orientando adequadamente o trabalhador quanto aos cuidados com a postura e a saúde, de modo a minimizar os fatores de risco de surgimento de doenças ocupacionais.

Para Zapparoli e Marziale (2006) a aplicação dos princípios da ergonomia, empreitada específica do fisioterapeuta do trabalho, pode propiciar uma interação adequada e confortável do ser humano com os objetos que maneja e com o ambiente de trabalho, aumentando assim o desempenho do trabalhador.

\section{CONCLUSÕES}

Através do estudo, conclui-se que a aceitação no meio empresárial de que a qualidade de vida do trabalhador está diretamente ligada a sua maior e melhor produtividade ainda gera dúvidas. Teixeira et al(2009) e Lacaz (2000) apresentam dois 
desafios para o mundo empresarial, o primeiro está relacionado à necessidade de uma força de trabalho saudável, motivada e preparada para a extrema competição existente, e o segundo é a capacidade da empresa responder a demanda de seus funcionários em relação a uma melhor qualidade de vida. Essas variáveis estão profundamente interligadas e induzem os empregadores a investir mais na implementação de programas de qualidade de vida.

Por tanto é perceptível a importância do profissional fisioterapêta no âmbito ocupacional, pois, através de suas habilidades e qualificações é possível proporcionar melhor qualidade de vida para o trabalhador, assim aumentando sua produtividade.

Por fim, percebe-se que apesar de tal importância do profissional e os benefícios proporcionados, ainda é lento o avanço e a aceitação dessa atuação em nossa realidade e que à necessidade de evolução, porém para que isso aconteça, faz-se necessário mais pesquisas sobre este assunto.

\section{BIBLIOGRAFIA}

SILVA, G. J. P. et al. Danos à saúde relacionados ao trabalho de fisioterapeutas que atuam em terapia intensiva. ASSOBRAFIR Ciência, v. 7, n. 2, p. 31-44, 2016.

OLIVEIRA, J. R. G. A importância da ginástica laboral na prevenção de doenças ocupacionais. Revista de Educação Física, v. 139, n. I, p. 40-49, 2007

Padula RS, Oliveira AB, Carregaro RL, Sato TO. Physical therapy in occupational health and ergonomics: practical applications and innovative research approaches. Braz. J.Phys. Ther. [Internet]. 2016; 20(5): 490-492.

FERNANDES, R.C.P et al. Repetitive tasks under time pressure: the musculoskeletal disorders and the industrial work. Revista Ciência e Saúde Coletiva, Rio de Janeiro, v.I5, n. 3, p. 931-42, mai. 2010.

Vialle LR, et al. Hérnia discal lombar. Revista brasileira ortopédica. São Paulo. 2010;45(I). Baú LM, Klein AA. O reconhecimento da especialidade em fisioterapia do trabalho pelo COFFITO e Ministério do Trabalho/CBO: uma conquista para a 
fisioterapia e a saúde do trabalhador. Brazilian Journal of Physical Therapy, 2009: $13(2)$.

ALVES, Henrique; OLIVEIRA, Izalvina; PEDRONI, Wemerson. Fisioterapia do Trabalho Nova Especialidade em Prova de Titulação. FisioBrasil, Laranjeiras, Edição no 96,p. 38-46, 2009.

TSUCHIYA, H.Z.C.; MENDONCA, C.S.L.; CESAR, A.C.G. Associação entre características pessoais, organização do trabalho e presença de dor em funcionários de uma indústria moveleira. Fisioter. Pesqui. ano 2009, vol.ı6, n.4, pp. 294-298.

Da Silva MC, Fassa AG, Kriebel D. Musculoskeletal pain in ragpickers in a southern city in Brazil. Am J Ind Med. 2006; 49:327-36.

OLIVEIRA, J.R.G. A prática da ginástica laboral. 3. ed. Rio de Janeiro. Ed. Sprint, 2006. Brazil AV, Ximenes AC, Radu AS, Fernandes AR, Appel C, Maçaneiro CH, et al. Diagnóstico e tratamento das lombalgias e lombociatalgias. Rev Bras Reumatol. 2004;66(6) 419-25.

Santos M. Hérnia de Disco: uma revisão clínica, fisiológica e preventiva. Revista digital. Buenos Aires. 2003;65.

Fernandes RCP, Carvalho FM. Doença do disco intervertebral em trabalhadores de perfuração de petróleo. Cad Saúde Pública. 200o;16(3):66ı-9.

LACAZ, F.A.C. Qualidade de vida no trabalho e saúde/doença. Ciênc. saúde coletiva, ano 2000, v.5, n.I.

Fernandes RCP, Carvalho FM. Doença do disco intervertebral em trabalhadores da perfuração do petróleo. Caderno de Saúde Pública. Rio de Janeiro. Mar.1994;16(3):115-26. 